\title{
EDITOR'S
}

COMMENT

\section{Radical Prostatectomy: Biochemical recurrence and prognostic factors}

The November-December 2013 issue of the International Braz J Urol presents original contributions with a lot papers in different fields. The papers come from many different countries such as Brazil, USA, Iran, France, Italy and Korea, and as usual the editor's comment highlights some papers. In this number we can observe some papers about prognostic factors and recurrence in patients submitted to radical prostatectomy. This surgery is perhaps the most interesting surgery for the urologist and this topic is always up to date.

Doctor Noronha and colleagues, from the Departments of Urology and pathology, from the state university of Campinas, Brazil performed on page 779 an interesting study in 400 patients about controversial clinicopathologic predictors of biochemical recurrence after radical prostatectomy and concluded that young patients and non-whites were not significantly associated with time to biochemical recurrence. The higher predictive value of Gleason score $4+3=7$ vs. $3+4=7$ discloses the importance of grade 4 as the predominant pattern. Most tumor extent evaluations on needle biopsies are predictive of time to biochemical recurrence however maximum percentage of cancer in all cores was the strongest predictor.

Doctor de Nunzio and colleagues from the University of Florence, Italy performed on page 793 a clinical study in prostatic cancer to explore the association between serum levels of Sex Hormone Binding Glo- bulin (SHBG) and the risk of developing prostate cancer (PCa) as well as high grade disease in men undergoing prostate biopsy and concluded that in this cohort of patients, serum levels of SHBG are not predictive of PCa or high grade disease. According to our experience SHBG should not be considered a biomarker in PCa diagnosis neither a marker for high grade disease.

Doctor da Silva and colleagues from the University of São Paulo Medical School, São Paulo, Brazil performed on page 800 an interesting study 


\section{EDITOR'S \\ COMMENT}

about the Single positive core in a prostate biopsy and this association with indolent prostate cancer and concluded that single core prostate cancer have clinically significant disease in the Radical Prostatectomy specimens, with considerable rates of overgrading for the Gleason Score, pT2c-pT3, extraprostatic extension, positive surgical margins and seminal vesicle invasion. The treatment plan must be evaluated individually for patients with single core PCa and must take into account other prognostic factors when determining whether a patient should be managed with active surveillance.

Doctor Tehranchi and colleagues from Urmia Urology and Nephrology Research Center, Urmia University of Medical Sciences, Urmia, Iran performed on page 832 a interesting randomized clinical trial about the effects of terazosin and tolterodine on ureteral stent discomfort and concluded that Terazosin plus tolterodine improves ureteral stent-related complications, including irritative symptoms, the amount of analgesics used, quality of life, flank pain and voiding pain but does not decrease obstructive symptoms or suprapubic pain.

Doctor Zambon and colleagues from the Federal University from São Paulo, São Paulo, Brazil and Wake Forest University, USA, performed on page 841 a study in a large population of Brazilian submitted to a health check up program and assess the uroflowmetry data and their correlation to IPSS and prostate size. The authors concluded that in asymptomatic men there is a weak correlation between IPSS, prostate size and uroflowmetric data. The establishment of different normal cutoffs seems to be complicated and uroflowmetry data should be interpreted with caution in order to avoid misdiagnosis.

Doctor Costa from Urogenital Research Unit of Rio de janeiro State University, Rio de Janeiro, Brazil, performed on page 853 a interesting basic research about the structure of the ureter in normal and anencephalic human fetuses and concluded that Fetuses with anencephaly showed the ureter with smaller diameter, area and thickness. These differences could indicate that anencephalic fetal ureters tend to have significant structural alterations, probably due to cerebral lesions with consequent brain control damage of ureter nerves. 


\section{EDITOR'S}

Doctor Costa and colleagues from Federal University of São Paulo, São Paulo, Brazil, performed on page 884 a interesting experimental research in about the histological characteristics of keratinized versus non-keratini- zed onlay island flaps in an experimental rabbit model. In this interesting study with application to hypospadias surgery the authors concluded that in this short-term rabbit model, we observed that the stratified squamous keratinized epithelium from the external prepuce kept its keratin production. There was no statistical influence of the flap type on the mean epithelial thickness.

Luciano A. FaVorito, MD, PhD

Associated Editor Internacional Braz J Urol 